Research Paper

\title{
Subtype Identification in Acutely Dissociated Rat Nodose Ganglion Neurons Based on Morphologic Parameters
}

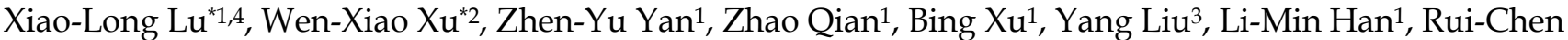

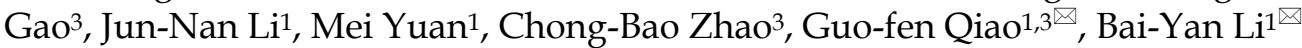 \\ 1. Department of Pharmacology, Harbin Medical University, Harbin, China \\ 2. Department of Orthopedics, the First Affiliated Hospital, Harbin Medical University, Harbin, China \\ 3. Key Laboratory of Cardiovascular Medicine Research, Ministry of Education, Harbin Medical University, Harbin, China \\ 4. Riley Heart Research Center, Division of Pediatric Cardiology, Herman B. Wells Center for Pediatric Research, Department of Pediatrics, \\ Indiana University School of Medicine, Indianapolis, USA \\ * These authors contributed equally to this work.
}

$\triangle$ Corresponding author: Dr. G-F Qiao (qiaogf88@yahoo.com.cn) or Dr. B-Y Li (baili@iupui.edu), Department of Pharmacology, Harbin Medical University, 157 Bao-Jian Road, Harbin 150081, China, 86-451-8667-1354

(C) Ivyspring International Publisher. This is an open-access article distributed under the terms of the Creative Commons License (http://creativecommons.org/ licenses/by-nc-nd/3.0/). Reproduction is permitted for personal, noncommercial use, provided that the article is in whole, unmodified, and properly cited.

Received: 2013.06.25; Accepted: 2013.07.01; Published: 2013.07.27

\begin{abstract}
Nodose ganglia are composed of A-, Ah- and C-type neurons. Despite their important roles in regulating visceral afferent function, including cardiovascular, pulmonary, and gastrointestinal homeostasis, information about subtype-specific expression, molecular identity, and function of individual ion transporting proteins is scarce. Although experiments utilizing the sliced ganglion preparation have provided valuable insights into the electrophysiological properties of nodose ganglion neuron subtypes, detailed characterization of their electrical phenotypes will require measurements in isolated cells. One major unresolved problem, however, is the difficulty to unambiguously identify the subtype of isolated nodose ganglion neurons without current-clamp recording, because the magnitude of conduction velocity in the corresponding afferent fiber, a reliable marker to discriminate subtypes in situ, can no longer be determined. Here, we present data supporting the notion that application of an algorithm regarding to microscopic structural characteristics, such as neuron shape evaluated by the ratio between shortest and longest axis, neuron surface characteristics, like membrane roughness, and axon attachment, enables specific and sensitive subtype identification of acutely dissociated rat nodose ganglion neurons, by which the accuracy of identification is further validated by electrophysiological markers and overall positive predictive rates is $89.26 \%(90.04 \%, 76.47 \%$, and $98.21 \%$ for A-, Ah, and C-type, respectively). This approach should aid in gaining insight into the molecular correlates underlying phenotypic heterogeneity of nodose ganglia. Additionally, several critical points that help for neuron identification and afferent conduction calibration are also discussed.
\end{abstract}

Key words: visualization, visceral sensory neuron, conduction velocity, whole-cell patch, action potential

\section{Introduction}

Visceral afferent nerves enable electrical signaling from their endings in internal organs to their soma

(first order neurons) in nodose ganglia. Thus, nodose ganglion neurons take center stage in relaying infor- 
mation from the peripheral to the central nervous system and investigation of their properties is of fundamental significance to a better understanding of the mechanisms regulating body homeostasis. It is well established that nodose ganglia house heterogeneous neuronal populations which are composed of myelinated A-, Ah-, and unmyelinated C-type neurons $(1,2)$. From a technical point of view, it is relatively straightforward to obtain high-quality electrophysiological recordings from isolated nodose neurons using the single-electrode patch-clamp approach, which is the preferred technique for membrane voltage and current measurements. However, the clear disadvantage of using isolated nodose neuron preparations lies in its inability to readily identify the neuron subtype based on conduction velocity measurements in the corresponding afferent fibers, simply because afferent fibers are lost during the enzymatic isolation procedure. In order to still be able to classify neuron subtypes in isolated cell preparations, several methods have been suggested, including measurement of neuron diameter (3), sensitivity to capsaicin (4), electrophysiological characteristics [e.g., hump over the course of repolarization (5), presence of tetrodotoxin-resistant (TTX-R) $\mathrm{Na}^{+}$channels (6). The presence of a repolarization hump has long been considered a specific feature of unmyelinated C-types in isolated neuron preparations $(5,7)$. However, our slice recordings show that humps can also be found in Ah-types (8), suggesting the possibility that myelinated Ah-types can be mis-classified as unmyelinated C-types in isolated preparations. Thus, caution should be used when employing the repolarization hump as sole criteria for unmyelinated C-types in isolated preparation. It should be combined with other electrophysiological and morphological features (see details below). In addition, the computer modeling study demonstrates that TTX-resistant (TTX-R) $\mathrm{Na}^{+}$ currents encoded in Nav1.8 is a responsible ion channel underlying the repolarization hump (9), therefore, the expression of a TTX- $\mathrm{R} \mathrm{Na}^{+}$current has been used to identify unmyelinated C-types in isolated nodose neurons (6), especially under experimental conditions that a priori preclude measurements of action potential properties to classify the neuron subtype. However, while we were developing the slice preparation, TTX-R Na+ currents were not only observed in unmyelinated C-type fibers (10) but also in myelinated Ah-type neurons (11). This particular fiber exhibited a CV exceeding $20 \mathrm{~m} / \mathrm{s}$ and persistence of an inward $\mathrm{Na}^{+}$current in the presence of $1.0 \mu \mathrm{M}$ TTX. Thus, expression of a TTX-resistant voltage-activated $\mathrm{Na}^{+}$current and fast conduction properties are not mutually exclusive, invalidating the prevailing assumption that C-type fibers can be iden- tified based on their TTX-resistivity.

In Addition, the expression of proteins [e.g., isolectin B4 (12), Transient Receptor Potential A1 (TRPA1) (13), BK-type $\mathrm{Ca}^{2+}$-activated-potassium channel (KCa1.1) (14)], and functional expression of hyperpolarization-activated current (Ih) through HCN $(15,16)$. However, these methods and markers are prone to erroneous interpretations, because they are influenced by animal age and gender $(1,2)$, time of the neurons in culture (17), and enzymatic digestions (see details in text). All these factors dramatically affect the power of the various classification approaches, which will be discussed in details in the text. Given on one hand the restriction of the single-electrode voltage-clamp technique to its use in isolated cells for measurements of fast and large transmembrane currents, and on the other hand given the limitations of existing approaches to identify subtypes of nodose neurons in isolated cell preparations, we sought to present an alternative algorithm which would enable us to identify subtypes of neurons isolated from nodose ganglia with high degrees of sensitivity and specificity. This algorithm utilizes microscopic structural patterns through visualization to classify nodose ganglia subtypes.

\section{Materials and Methods}

All images were collected using an upright microscope (Axioskop, Zeiss) equipped with a 40x 0.8 NA water immersion lens, fluorescence detection components, and a digital camera system (AxioCam, Zeiss). Following image collection, transmembrane action potentials were recorded from isolated or in situ nodose neurons using the patch-clamp technique in whole-cell current-clamp mode. In case of in situ preparations, vagal stimulation-evoked action potentials were recorded from the soma, and action potential (AP) properties were determined. Both neonatal and adult Sprague-Dawley (SD) rats of either gender were used. All animal use protocols were approved by the Institutional Animal Care and Use Committee of the School of Medical Science, Harbin Medical University (Harbin, China).

\section{Primary culture of isolated neonatal nodose neurons}

The procedures for dissection and enzymatic isolation of nodose ganglion neurons have been reported previously (7). Briefly, on the day of experiments, whole nodose ganglia from at least two 5- to 7-day-old rat pups were excised bilaterally and placed in a chilled $\left(\sim 4^{\circ} \mathrm{C}\right)$ nodose complete media $(\mathrm{NCM})$, consisting of Dulbecco's Modified Eagle's F-12 (Invitrogen), 5\% fetal bovine serum (HyClone), 0.01\% penicillin-streptomycin (Sigma), and 0.1\% $\mathrm{MITO}^{\mathrm{TM}}+$ 
serum extender (BD Biosciences). The ganglia were digested using trypsin-3X (5 mg/ml, Worthington) for $30 \mathrm{~min}$ at $37^{\circ} \mathrm{C}$. The enzyme solution was replaced with NCM and ganglia were gently titrated with an aspiration pipette. The isolated cells were plated on poly-D-lysine - coated cover slips $(10 \times 10)$ and stored in $5 \% \mathrm{CO}_{2}+$ air in a humidified chamber until use.

\section{Primary culture of isolated adult nodose neu- rons}

All procedures have been described in details previously (8). Briefly, both nodose ganglia were excised from adult rats of at least 14 weeks of age and immediately placed into chilled $\left(\sim 4^{\circ} \mathrm{C}\right)$ recording solution. The connective tissue around the ganglia was carefully removed and each ganglion was cut into 6 small pieces under a surgical microscope $(40 \times)$. The ganglion tissue was transferred to enzyme solution (Earle's balanced salt solution, Sigma) containing 20 units/ml of Papain in a 15-ml centrifuge tube, digested at $37^{\circ} \mathrm{C}$ in $5 \% \mathrm{CO}_{2}$ - air in a moisturized chamber for $20 \mathrm{~min}$, and then transferred into a $2^{\text {nd }}$ enzyme solution (Earle's balance salt solution, Sigma) containing $1.0 \mathrm{mg} / \mathrm{ml}$ type II collagenase and 2.0 $\mathrm{mg} / \mathrm{ml}$ Dispase for 30-min incubation under the same conditions. After digestion, the enzyme solution was completely removed and $1.0 \mathrm{ml}$ of warm $\left(\sim 36{ }^{\circ} \mathrm{C}\right)$ $\mathrm{NCM}$ with $1.0 \mathrm{mg} / \mathrm{ml}$ of albumin (Sigma) was added and the ganglia were gently triturated with a glass aspiration pipette until they were no longer visible. The cell suspension was then transferred onto poly-D-lysine - coated cover class $(10 \times 10)$ and incubated for at least $60 \mathrm{~min}$ to allow neurons to attach to the glass surface. Finally, 2 to $3 \mathrm{ml}$ of warm NCM was added to the culture dish and neurons were incubated for another 2-3 $\mathrm{h}$ before use, enabling their recovery from enzymatic digestion.

\section{Vagus-nodose slice preparation}

Adult male or female Sprague-Dawley rats (220 - $250 \mathrm{~g}$, at least 14 weeks of age) were used for the preparation of nodose slices. Bilateral dissection of the nodose ganglia with $\sim 2.0-\mathrm{cm}$ segments of the vagus nerves still attached, slicing, and enzymatic digestion have been described previously (8). Briefly, surgical dissection of the nodose ganglia was performed under a stereomicroscope $(40 \times)$. The nodose ganglia were placed immediately in chilled $\left(\sim 4{ }^{\circ} \mathrm{C}\right)$ recording solution, all connective tissue around ganglia and nerve was completely removed, and the ganglia were sliced without damaging the vagus nerve. Following the addition of $1.0 \mathrm{mg} / \mathrm{ml}$ type II collagenase (Worthington), the ganglia slices with the attached vagus nerve segment were incubated at $37{ }^{\circ} \mathrm{C}$ for 30 min followed by fresh support medium with 5.0 $\mathrm{mg} / \mathrm{ml}$ trypsin (Worthington) at $37{ }^{\circ} \mathrm{C}$ for an additional 20-25 min, depending upon animal age. The enzyme solution was then completely replaced with recording solution prior to transfer of the ganglia to the recording chamber.

As described previously (8), the nodose ganglion slices with the attached vagus nerve segment were transferred to the recording chamber with the cut surface facing up and held in position by a harp, preventing tissue movement during recording and ensuring uninterrupted exposure of the cut surface to the recording solution. For CV measurements, a bipolar stimulation electrode was placed at the distal end of the vagus nerve segment, ca. $15 \mathrm{~mm}$ from the recording electrode positioned close to the ganglion. All above procedures are completed before electrophysiological recording was begun. To examine the conduction property of the truncated vagus nerve, short-duration $(\leq 200 \mathrm{~ms})$ monophasic constant current pulses were applied via the bipolar stimulation electrode. Pulse durations exceeding $200 \mathrm{~ms}$ may cause interference with the evoked electrical signal recorded close to or inside the ganglia, and should be avoided. To examine the frequency response of the preparation, trains of electrical stimuli $(1-100 \mathrm{~Hz})$ were delivered to myelinated A- and Ah- afferent fibers, and unmyelinated C-type visceral afferent fibers using a programmable stimulator (Master- 8 , the 8-channel programmable Pulse Generator, AMPI, Jerusalem, Israel).

\section{Critical techniques and limitations for intact nodose slice preparation}

Slice preparations of vagus-nodose ganglia were first established by us a decade ago (8) using adult rats. The advantages and disadvantages of this preparation have also been discussed in our previous work (8), but some additional technical details had not been made available previously. First, after slicing and proper treatment with enzymes, the slice has to be placed into the recording chamber with the cutting surface up (Supplementary Material: Figure. S1B); this is very important, since the treated tissue becomes very sticky, and care must be executed not to damage afferent, especially unmyelinated fibers. If the slice is placed properly, the cutting surface becomes less reflective and cells located within different layers remain in different focal planes compared with those neurons from untreated area of slice (Supplementary Material: Figure S1A), that would facilitate patching of the cells under microscopic guidance. Second, the first three layers of neurons remain accessible for electrophysiological recordings only with proper enzyme treatment and correct orientation of the slice in the perfusion chamber. Third, with this preparation, 
the superficial three layers of cells remain accessible for patching. The first and second layer of cells is easy to reach for patching; while a higher positive intrapipette pressure has to be applied to reach the third layer. Importantly, the second and third layers of cells typically provide higher-quality recordings because their membranes are less damaged by enzymes compared to the cells at the surface. Clearly, the advantages of the slice preparation are significant; however, the limitation with this preparation with regard to measurements of the fast activated $\mathrm{Na}^{+}$ current should be discussed. Because of its large size and fast activation (relative to the time constant for charging the membrane capacitor), voltage-gated $\mathrm{Na}^{+}$ currents are notoriously difficult to record, even when electronic capacitance and resistance compensation are applied to improve voltage-control. Loss of adequate voltage control results in erroneous measurements of both $\mathrm{Na}^{+}$current amplitude and activation kinetics (Supplementary Material: Figure S2B and C). Another disadvantage of slice preparations is the limited and/or delayed access of individual cells to exogenous chemicals, neurotransmitters, and ion channel agonists/antagonists. For example, it may take a long time for an externally applied compound to diffuse to a second- or third-layer neuron, delaying manifestation of its electrophysiological effect. Thus, caution and more patience have to be paid during slice recordings.

\section{Aortic depressor nerve labeling}

In order to confirm the similarity of the electrophysiological characteristics between general population of vagal ganglion neurons and functional-specific population of baroreceptor neurons with myelinated and unmyelinated fiber types, the aortic depressive nerve (ADN) was fluorescently labeled with Dil in a cohort of adult female rats at least 2 weeks prior to electrophysiological experimentation. Younger rats ( 75-100 g, at least 4 week of age) are preferred for nerve labeling because of less connective tissue around ADN. The procedure of Dil labeling was identical to that described in details previously (18). Briefly, under general anesthesia, the left ADN was dissected free at a 2-cm distance from the nodose ganglion. Dil crystals were placed on top of the ADN and then completely sealed to prevent Dil molecules from leaking onto the vagus. The wound was closed and antibiotics were applied locally. After 10 weeks, DiI-treated animals were sacrificed, nodose ganglia were removed, processed for electrophysiological experimentation as described above, and baroreceptor neurons were dually identified via both conducting properties and fluorescence.

\section{Further confirmation of microscopically clas- sified isolated afferent neurons}

Microscopic classification of individual isolated neurons as belonging to A-, Ah- or C-type fibers was confirmed by applying electrophysiological criteria that had been established previously (1). Briefly, to be classified as myelinated A-type nodose neuron, the AP firing threshold (APFT) must be more negative than $-40 \mathrm{mV}$, the maximal upstroke velocity (UVMAX) must exceed $250 \mathrm{mV} / \mathrm{ms}$, and AP duration (APD 50 ) must be less than $1.0 \mathrm{~ms}$ with no repolarization hump. The same criteria for APFT and $\mathrm{UV}_{\mathrm{MAX}}$, but an $\mathrm{APD}_{50}$ $>1 \mathrm{~ms}$ with a detectable repolarization hump, were considered diagnostic for myelinated Ah-type nodose neurons. For unmyelinated C-type fibers, APFT must be less negative than $-35 \mathrm{mV}, \mathrm{UV}_{\mathrm{MAX}}$ must not exceed $250 \mathrm{mV} / \mathrm{ms}$, and $\mathrm{APD}_{50}$ must be $>2 \mathrm{~ms}$ with a detectable repolarization hump. In addition, the cells' current responses to external application of $\alpha \beta$-methyl-ATP and capsaicin were used as pharmacological classification criteria.

\section{Electrophysiological patch recordings}

Whole-cell recordings were performed using the Axopatch 700B amplifier (Axon Instruments, Union City, CA, USA). Borosilicate glass pipettes (Sutter Instruments) with a tip resistance ranging from 1.5-1.8 $\mathrm{M} \Omega$ were used, as measured in AP recording solution (see below). Following the formation of a G $\Omega$ seal, the pipette capacitance was compensated. The total cell capacitance $(40-70 \mathrm{pF})$ and electrode access resistance (3-5 M $\Omega$ ) were also compensated by $60-80 \%$. All experiments were conducted at room temperature $\left(22-23{ }^{\circ} \mathrm{C}\right)$. Data traces were low-pass filtered at 10 $\mathrm{kHz}$ and digitized at $50 \mathrm{kHz}$ with 16-bit resolution using pCLAMP 10.2 (Axon Instruments) and Digidata 1440A (Molecular Devices, Sunnyvale, CA, USA) operating on a PC platform.

\section{Recording protocols}

For current-clamp experiments, two distinctly experimental protocols were executed. First, a single somatic AP was elicited by applying a brief $(\leq 2.0 \mathrm{~ms})$ suprathreshold positive current pulse through the patch electrode. Second, depolarizing current steps (50 - $300 \mathrm{pA})$ of 1-s duration from a holding potential of $-60 \mathrm{mV}$ were applied to evoke repetitive action potentials. For voltage-clamp experiments, the recording protocol is described in details in the results section.

\section{Recording solutions}

For action potential (AP) recordings, the composition of the intracellular solution was (in $\mathrm{mM}$ ): $\mathrm{NaCl}$ 10; $\mathrm{KCl} 50 ; \mathrm{K}_{2} \mathrm{SO}_{4} 50 ; \mathrm{MgCl}_{2}$ 5.0; HEPES 10.0, $\mathrm{pH}$ adjusted to 7.25 using $1 \mathrm{~N} \mathrm{KOH}$. Immediately prior to 
filling the patch pipettes, $2.0 \mathrm{mM} \mathrm{Mg}$-ATP (Sigma) and Na-GTP $2.0 \mathrm{mM}$ (Sigma) were added to the pipette solution, along with $4.0 \mathrm{mM}$ BAPTA-K and 0.25 $\mathrm{mM} \mathrm{CaCl}_{2}$ for a final buffered $\left[\mathrm{Ca}^{2+}\right]_{\mathrm{i}}$ of $100 \mathrm{nM}$. The composition of the extracellular recording solution was (in $\mathrm{mM}$ ): $\mathrm{NaCl} 137 ; \mathrm{KCl} 5.4 ; \mathrm{MgCl}_{2} 1.0 ; \mathrm{CaCl}_{2} 2.0$; glucose 10; HEPES 10, $\mathrm{pH}$ adjusted to 7.30-7.35 using $1.0 \mathrm{~N} \mathrm{NaOH}$. The osmolarities of the extracellular and intracellular solutions were adjusted to 310-315 and 290-295 mM/kg, respectively, using D-manitol (Sigma).

\section{Data analysis}

Pooled statistics were calculated using Excel (Microsoft, Bellevue, WA, USA) with measures expressed as the mean $\pm 1 \mathrm{SD}$. Comparisons between populations were performed using Student's $t$-test or ANOVA where appropriate. Data populations exhibiting an overlap of $5 \%$ or less were considered to be significantly different.

\section{Results \& Discussion}

\section{Neuronal soma diameter and whole-cell ca- pacitance}

In the literature, isolated nodose neurons have been classified based on diameter and/or action potential properties. For example, the presence of a repolarization hump or expression of certain voltage-gated $\mathrm{Na}^{+}$channel isoform has been considered diagnostic for unmyelinated C-types. In contrast, our data does not confirm the utility of soma diameter as a criteria to distinguish between neuron subtypes in nodose ganglia, because we found no significant difference for the diameter between myelinated A-type and unmyelinated C-type nodose neurons (Figure. 1, Table 1). Similarly, the magnitude of whole-cell capacitance (WCC), which is directly proportional to the outer membrane area, was similar between A-type and C-type nodose neurons, both in ganglia from adult and neonatal rats (Table 1). Thus, identification of fiber types based on soma diameter or capacitance is not appropriate. However, the soma diameter and WCC of nodose neurons significantly increased during postnatal development (Table 1). The soma diameter may also change in response to culture time (Table 2), i.e., showing a more ball-shape/smaller in diameter cultured for shorter time (1-3 h) and more flattening/larger in diameter cultured for a longer time (8-12 h, Table 2), further confounding the utility of cell soma diameter as a neuron subtype classifier. This observation is different from published data with DRG neurons (17) that become smaller in diameter along with 72-96 h culture, whereas nodose neurons from our study are only cultured for $12 \mathrm{~h}$.
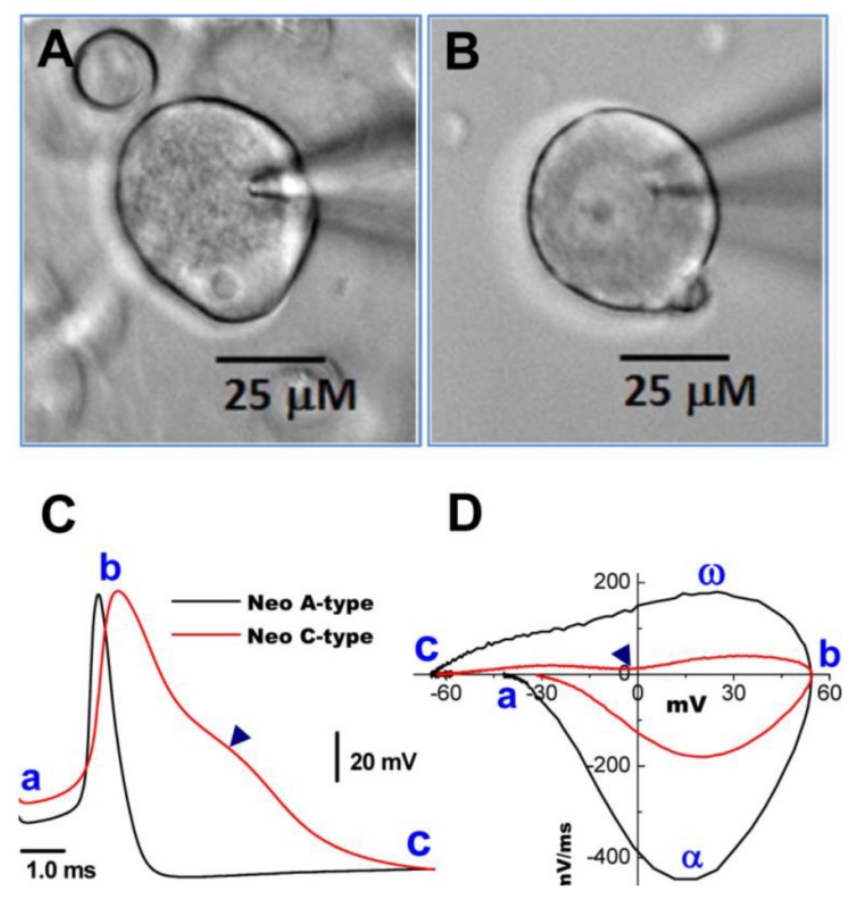

Figure I: Images of myelinated A- and unmyelinated C-type nodose neurons isolated from neonatal rats. A: A-type; B: C-type. Neonatal nodose neurons were cultured for less than 10 hours; $C$ : action potentials (AP) recorded from the same neurons shown in (A) and (B); $D$ : phase plots created from APs shown in (C); a - c: indicate the AP firing threshold, the peak of AP, and the voltage minimum of the after-hyperpolarization (AHP), respectively; $\alpha$ and $\omega$ indicate maximal upstroke velocity/total inward currents and maximal downstroke velocity/total outward currents, respectively. ( $\Delta)$ in (C) and (D) indicates voltage at which the repolarization hump occurs.

Table I: Comparison of whole-cell capacitance and soma diameter of nodose neurons isolated from adult and neonatal rats. Average data are presented as mean $\pm \mathrm{I} S \mathrm{SD}, \mathrm{n}=$ number of recordings. $* P<0.05$ and $* * P<0.01$ vs. adult.

\begin{tabular}{llll}
\hline & \multicolumn{3}{c}{ Nodose neurons isolated from adults } \\
Type $(\mathrm{n})$ & A- $(51)$ & Ah- $(32)$ & C- $(107)$ \\
WCC $(\mathrm{pF})$ & $66.2 \pm 6.01$ & $58.8 \pm 3.98$ & $62.1 \pm 5.03$ \\
Diameter $(\mu \mathrm{m})$ & $57.6 \pm 8.23$ & $52.5 \pm 4.72$ & $54.8 \pm 6.08$ \\
& \multicolumn{4}{c}{ Nodose } \\
Type $(\mathrm{n})$ & A- $(44)$ & Ah- $(29)$ & C- $(102)$ \\
WCC $(\mathrm{pF})$ & $35.1 \pm 4.3^{* *}$ & $33.2 \pm 3.1^{* *}$ & $34.7 \pm 5.5^{* *}$ \\
Diameter $(\mu \mathrm{m})$ & $37.0 \pm 2.31^{*}$ & $35.6 \pm 1.21^{*}$ & $36.0 \pm 1.05^{*}$ \\
\hline
\end{tabular}

Note: WCC: whole-cell capacitance. 
Table 2: Size changes in vagal ganglion neurons along with the culture time. Adult isolated vagal ganglion neurons were cultured and individual neuron size was continuously monitored. In this observation, isolated neurons were plated directly on the bottom of Petri dish and the neurons within the pre-marked area $(5 \times 5$ $\mathrm{mm}$ ) were observed at different time points with images taken at the same time for later measurements. Data were averaged from two pre-marked areas of each culture and total 5 cultures were investigated. Pooled data were presented as mean $\pm \mathrm{I} S \mathrm{SD}, \mathrm{n}=$ number of neurons observed. $* P<0.05$ and $* * P<0.0$ I vs I hour; $\dagger P<0.05$ vs 3 hours.

\begin{tabular}{|c|c|c|c|}
\hline \multicolumn{4}{|c|}{ Neuron size changes in diameter $(\mu \mathrm{m})$} \\
\hline & $\mathrm{n}=76,1 \mathrm{~h} \mathrm{n}=68,3 \mathrm{~h}$ & $\mathrm{n}=72,12 \mathrm{~h}$ & ANOVA \\
\hline $\mathrm{X}$ axis & $54.3 \pm 4.4259 .1 \pm 4.63^{*}$ & $65.5 \pm 4.03^{* *}+65.7 \pm 3.88^{* *}$ & $\mathrm{P}<0.05$ \\
\hline Y axis & $4.48 \pm 0.243 .01 \pm 0.19^{*}$ & $2.17 \pm 0.16^{* *} \dagger 2.14 \pm 0.15^{* *}$ & $\mathrm{P}<0.05$ \\
\hline
\end{tabular}

\section{Microscopic characterization of nodose neu- rons isolated from neonatal rats}

Neurons isolated from nodose ganglia of neonatal rats are widely used for electrophysiological studies because at this age their properties are not influenced by the gender with easy preparation. Thus, a simple and reliable method to identify subtypes of nodose neurons would be of great benefit to the research community. Based on our long experience with this preparation, we hypothesized that distinct differences in the microscopic appearance of the cell surface between myelinated A- and unmyelinated C-type VGNs may aid in distinguishing neuronal subtypes. Representative examples are shown in Figure 1 . Images were taken at $400 x$ magnification using a water immersion objective. The soma of a myelinated A-type neuron (Figure 1A) exhibited a rougher surface and a more peripheral location of the nucleolus compared with the soma of an unmyelinated C-type fiber (Figure 1B). The neurons identified by these visualized characteristics could further be confirmed by transmembrane action potential recordings and the phase plots that revealed marked differences in the electrical properties of A- and C-type fibers (Figure $1 C$ and D) (7).

However, for current measurements using the single-electrode voltage-clamp approach, it remains a technical challenge to identify the neuron subtypes in isolated preparations, i.e. without knowledge of the afferent conduction velocity. Current measurements in slice preparations, specifically measurements of large, fast activating voltage-dependent $\mathrm{Na}^{+}$currents, are associated with technical limitations as discussed in methods. Thus, adult isolated neurons would be indispensable for the study of voltage-gated $\mathrm{Na}^{+}$ channel function, both under physiological and disease condition. From clinical points of view, the ion channel functions and its molecular expressions are extremely important for neuronal excitability of visceral afferent neurons in nodose. The ontogeny in ion channel expression has been well demonstrated in nodose neurons $(19,20)$ and sympathetic neurons (21). This developmental change in ion channels would definitely influence the baroreceptor/baroreflex function $(22,23)$. Therefore, animal models established using adult nodose neurons both in situ and in vitro are closely mimicking the human disease condition, such as chronic heart failure (24), diabetes (25), and hypertension (26). Accordingly, alternative approaches to identify the neuronal subtype in isolated preparations would be extremely useful for voltage-clamp measurements. In some cases, for example, certain type of neuron needs to be a focused and toxin as channel modulator needs to be applied, then visual prediction of neuron subtype prior to patching would be definitely greater benefit to investigator than the electrophysiological identification (1) after patch collection of action potential.

\section{Microscopic structural characterization of nodose neurons isolated from adult rats}

With the proper enzyme treatment and culture conditions as described above, a larger percentage of myelinated A-type nodose neurons were found to exhibit short segments of myelinated fibers still attached to the soma (Figure 2A) compared with unmyelinated C-types in the same preparation (Figure 2B). The attached segments with significant nodes are easily distinguished from neuronal satellite cells because of their numbers, shape, and diameters (27), which, in sensory ganglia, these neuronal satellites cells ( 1 or 2 identified by glial marker glutamine synthetase) form a thin layer (less than $2 \mu \mathrm{m}$ ) with connective tissue around neurons. More importantly, this thin layer of satellite cells is tightly attached with neuron cell body and unable to be seen under low power microscope with specific marker, however, the segment of myelinated fiber (Figure 2A) has only one end attached to the cell body and the other end floated, which is easy to recognized during the extracellular perfusion. Papain treatment seems to play a key role in this process because the same phenomenon is observed on almost all neurons in culture without Papain treatment. The neuronal subtype was further confirmed via AP recordings (Figure $2 \mathrm{C}$ ) and generation of phase plots (Figure 2D). 

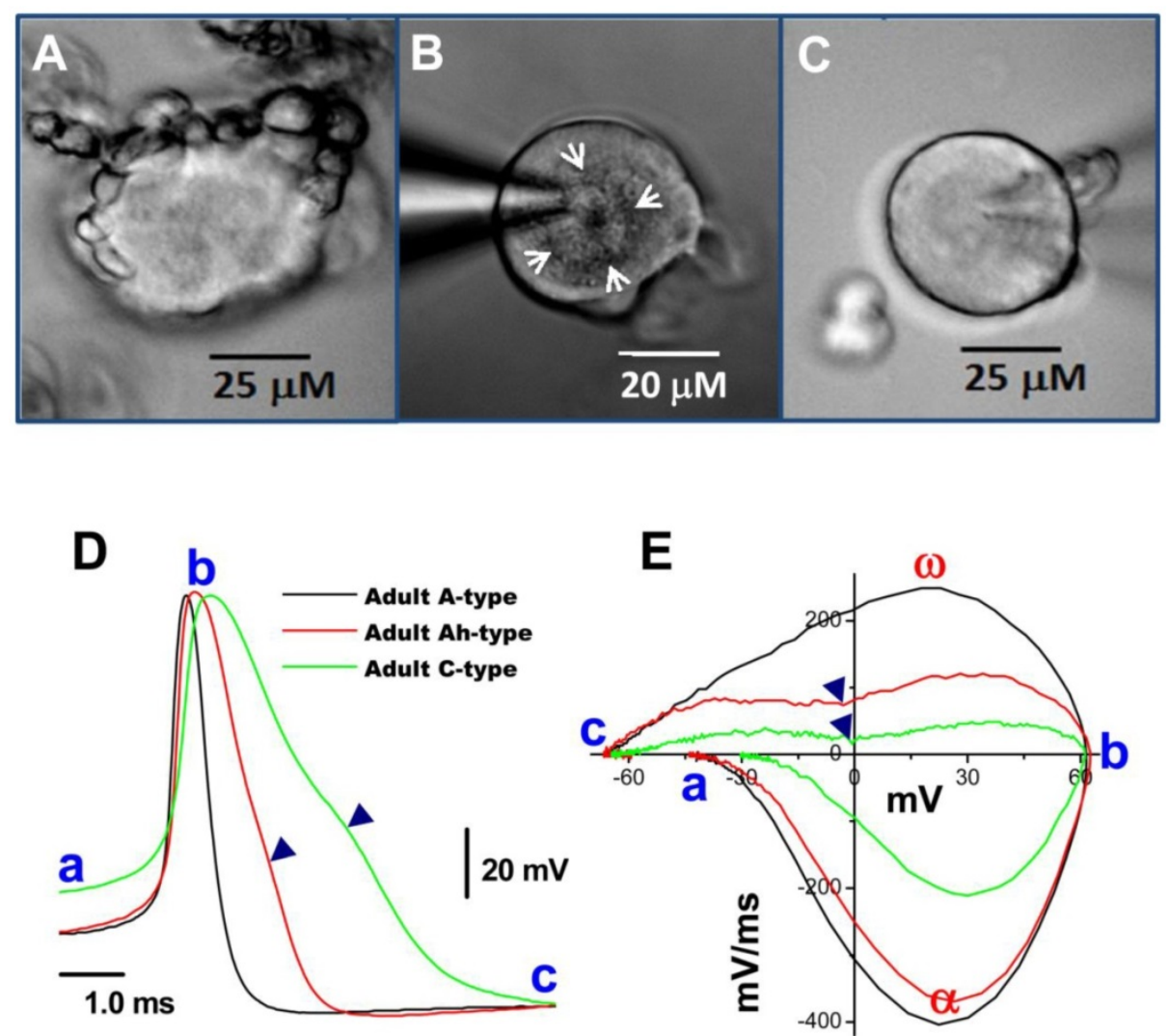

Figure 2: Bright field images of myelinated A- and unmyelinated C-type nodose neurons isolated from adult rats. A: A-type; B: Ah-type; C: C-type. Adult nodose neurons were cultured for less than 10 hours; D: APs recorded from the same neurons shown in (A), (B) and (C); E: phase plots created from APs shown (A)-(C); in the phase plot, $a-c$ indicate the APFT, the peak of AP, and the minimum voltage of the AHP, respectively; $\alpha$ \& $\omega$ indicate points of maximal upstroke velocity, e.g. total inward currents and downstroke velocity, e.g. total outward currents, respectively. ( $\nabla)$ in either (D) \& (E) indicates the voltage at which the repolarization hump occurs. White arrows indicate regions of roughed membrane appearance seen in Ah-type neurons in culture.

\section{Microscopic structural identification of mye- linated Ah-type afferent neurons isolated from nodose ganglia of adult female rats}

In most instances, wherein isolated preparations were used for voltage-clamp protocols, AP and electrophysiological makers read from AP, such as APFT, $\mathrm{APD}_{50}, \mathrm{UV}_{\mathrm{MAX}}$, and the repolarization hump, were not available; therefore microscopic characters became more important in identification of neuron types, especially Ah-types. The following microscopic criteria allowed a $70 \%$ likelihood of correctly identifying these neurons (Figure 2B, white arrows): (1) a rough membrane appearance restricted to the cell center. This is significantly different from myelinated A-type neurons that typically show rough appearance of the entire visible portion of the cell surface; (2) Near perfectly round shape with equal length of long axis and short axis; (3) Ah-type neurons exhibit relatively less variation in soma diameter $(\sim 50-60 \mu \mathrm{m})$ and whole-cell capacitance $(\sim 60 \mathrm{pF})$ in adult female rats, while the diameter and whole-cell capacitance of A-type neurons vary significantly $(30-70 \mu \mathrm{M}$ and 25-80 pF); (4) Parts of axons may still be attached to the neurons if the preparation was not enzymatically overdigested. Among there microscopic characteristics, centrally rough membrane and nearly round ship are keys for distinguish Ah-type from others; whereas, the diameter and axon attachment are considerable help in identification process (Table 3). However, there is a remaining probability of misidentifying an Ah types as an A- or C-type neurons (Table 5), suggesting Ah-type neurons share some features with both A-type (fast CV) and C-type (repolarization Hump). Alternatively, pharmacological approaches may further help Ah-type verification and in the following circumstances, both A- and C-types would be excluded: (1) without expression of TTX-R component or/and capsaicin-positive In case of $\mathrm{Na}^{+}$current recordings because A-type dose not express TTX-R and only C-type is positive to capsaicin $(1,10)$; $(2)$ without expression IbTX-sensitive component or/and Capsaicin-positive in case of $\mathrm{K}^{+}$current recording simply because A-type do not express BK-KCa channel and only C-type responds to capsaicin $(1,14)$. However, there is still no better way to exclude A-type during $\mathrm{Ca}^{2+}$ current recordings because both A- and Ah-types functionally express $\mathrm{N}$-type $\mathrm{Ca}^{2+}$ channel (28). If there 
is an opportunity to record action potentials from those isolated cells, their characteristics (Figure 2D \& E) would match those of myelinated Ah-type fibers, including low APFT $(<-40 \mathrm{mV}$; Figure 2D-a, 2E-a), faster upstroke velocity ( $>250 \mathrm{mV} / \mathrm{ms}$; Figure 2E-a), narrow action potential duration $(<2.0 \mathrm{~ms}$; Figure $2 \mathrm{D})$, and distinct hump ( $\boldsymbol{\nabla})$ during repolarization (Figure 2D \& E).

\section{Microscopic structural characterization of nodose neurons from slice preparation}

Occasionally, conduction velocity cannot be assessed during electrical vagal stimulation for several reasons. First, fibers can be damaged during the preparation. Interestingly, however, in these cases one may still be able to identify the fiber type by microscopic appearance of the cell soma and cell surroundings. Myelinated A-type nodose neurons are usually surrounded by a larger fiber (Figure 3A, indicated by blue arrows) with nodes that can be removed via application of a positive pressure to the pipette interior, while unmyelinated C-types are typically observed to have a tiny unmyelinated fiber attached to them (Figure 3B, indicated by single white arrow), which can be seen by gently pulling the electrode away after getting a tight seal. All these observations were confirmed by recordings of action potential elicited by electrical vagal nerve stimulation (Figure 3C) or soma stimulation with brief pulses delivered via the recording electrode. The current phase plot (the derivative current change plots as the function of membrane voltage) is another useful analysis tool to look into more details of total currents over the voltage change during the depolarization (negative loops) and repolarization (positive loops) phase, enabling assessment of differences between myelinated and unmyelinated neurons (Figure 3D). In addition, it is worth mentioning that the cell body becomes more ball shaped compared with a tightly attached cell (Figure 3B) when it is separating from the slice (Figure 3A \& B).

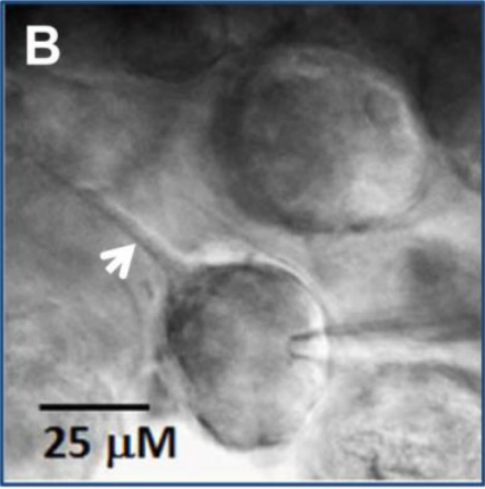

C

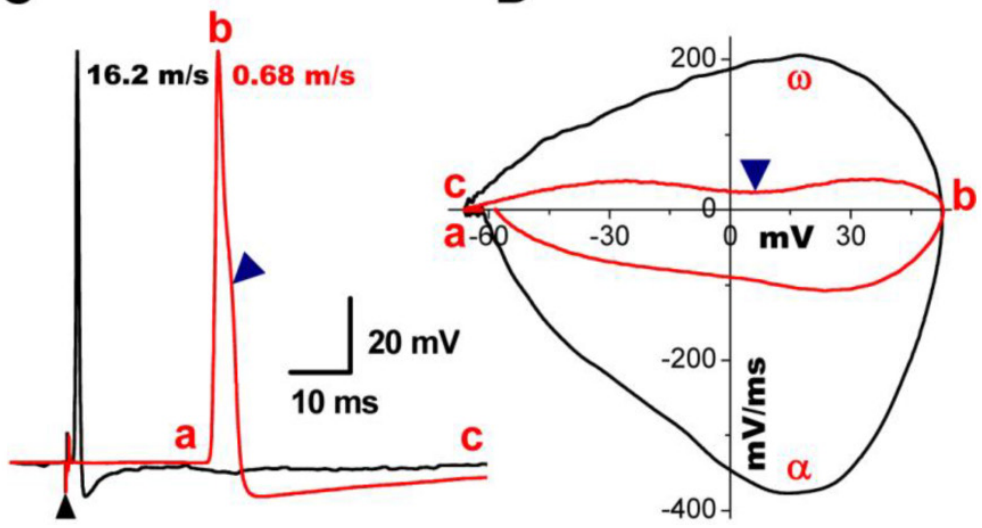

Figure 3: Bright field images of myelinated A- and unmyelinated C-type nodose neurons from intact slice preparation of adult rats. A: A-type neuron surrounded by a large-diameter (> $5 \mu \mathrm{M}$ ) myelinated fiber (arrows); B: C-type neuron with unmyelinated dendrite indicated by arrow. C: vagal stimulation-evoked APs recorded in the same neurons in $(A)$ and $(B)$. ( $\mathbf{A})$ indicates the repolarization hump. Afferent fiber conduction velocities (CV) were calculated for further identification of neuronal subtypes. D: Phase plot created from APs shown in (C), a - c: indicate the APFT, the peak of AP, and the minimum voltage of AHP, respectively; $\alpha$ \& $\omega$ indicate maximal upstroke velocity, e.g. total inward currents and downstroke velocity, e.g. total outward currents, $(\boldsymbol{\Delta})$ indicates the location of repolarization hump. 


\section{Identification of baroreceptor neurons in slices from adult rats following Dil labeling}

In situ DiI labeling can also aid in identifying subtypes of baroreceptor neurons either in isolated or slice preparations as demonstrated in Figure 4. Using the slice preparation of adult female rat, the subpopulation of myelinated Ah-type baroreceptor neuron was also observed (Figure 4A) with attached nodded fibers (white arrow) that was also identified by its conduction velocity (Figure 4B) and waveform character shown on the phase plot (Figure $4 \mathrm{C}$ ), such as the maximal depolarization $(\omega)$ and the hump over the time course of repolarization $(\mathbf{\Delta})$. The myelinated Ah-type neuron is characterized by faster conduction velocity (Figure 4B and Supplementary Material: Figure S2A) and repolarization hump (Figure 4C, $\mathbf{\Lambda}$ ), a characteristics of classical A- (Figure 4A, black trace) and C-type neuron (Figure $4 C \& D$, red trace and indicated by $\boldsymbol{\nabla}$ ), respectively. Just due to the AP repolarization hump, Ah-type neurons may be easily and mistakenly sorted into unmyelinated C-types. This could also be one of the reasons why in previously studies some neurons identified as C-types do not respond to capsaicin $(29,30)$ but $\alpha \beta-m-A T P$. In this situation, checking some more characters of the AP firing threshold and upstroke velocity and comparing visualized appearance of isolated neurons would give a great help to distinguish the fiber types.

For identifying the neuron type and its sensory modality, such as a baroreceptor neuron, at the same time, Dil labeling for at least one week (necessary for the lipophilic dye to transport to the nodose ganglia) prior to electrophysiological experimentation is a feasible approach. It is more practical to use young rats of less than $100 \mathrm{~g}$ bodyweight for labeling. The key point here is to avoid Dil contamination of the vagus nerve. The combination with fluorescence labeling, microscopic appearance and electrophysiological phenotype will enable correct neuron classification even though when the slice preparation is used.

\section{Afferent fiber distortion and conduction ve- locity correction}

It has been well documented $(7,8,16,31-33)$ that the conduction velocity of visceral afferent fibers varies from 2 to more than $20 \mathrm{~m} / \mathrm{sec}$ at room temperature. The reasons for this variation have remained elusive. We have found that vagal nerve fibers retracted markedly along their longitudinal axon during enzymatic treatment (Figure 5), most likely because of connective tissue shrinkage in- and outside the nerve bundle, despite careful mechanical removal of all visible connective tissue before tissue processing. Actual nerve path length between stimulus and recording electrode is significantly longer than the measured distance between them, causing artificially low magnitudes of conduction velocities. Typical examples of axonal shrinkage for large-, intermediate- and small-diameter axons following enzymatic treatment are shown in Figure 5A-F, respectively. The calibration factors, utilizing a geometrical correction, were $0.73,0.68,0.66,0.69,0.75$, and 0.75 , respectively. Average data of conduction velocity before and after correction were significantly different $(\mathrm{P}<0.001, \mathrm{n}=$ 12 slice preparations) (Table 4).

Animal age also affects conduction velocity, because myelination is not complete until 12-14 weeks of age. Thus, slices prepared from rats of less than 12 weeks of age would be expected to exhibit reduced nerve conduction velocities (31) For example, aortic depressor nerve axons are less number of myelinated afferents in 50-day-old rats of either gender (Supplementary Material: Figure S3) compared with adults (1).
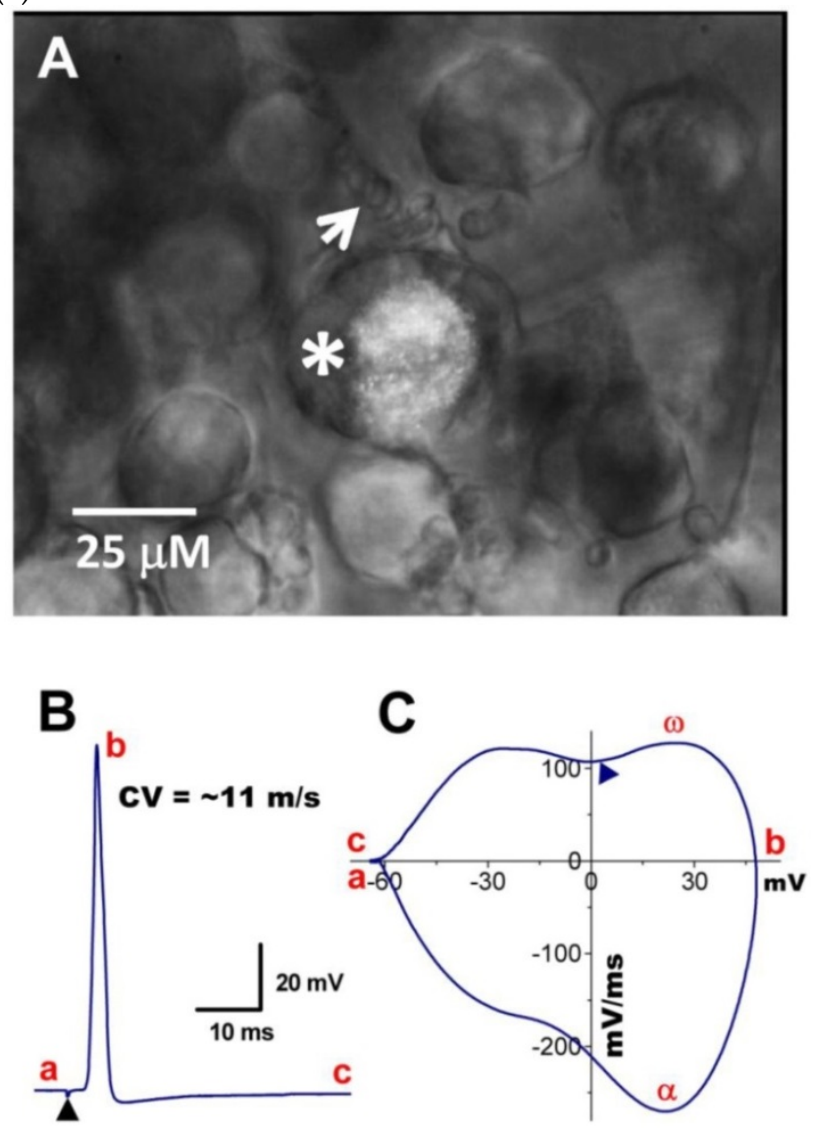

Figure 4: Myelinated Ah-type baroreceptor neurons from a slice preparation. A: Dil-labeled Ah-type baroreceptor neuron (asterisk) with nodded myelinated fiber (white arrow) attached to its cell body; B: APs evoked by vagal stimulation $(\boldsymbol{\Delta})$ with conduction velocity $>10 \mathrm{~m} / \mathrm{sec}$; $C$ : phase plot generated from the AP shown in (B). A repolarization hump is visible near zero voltage $(\boldsymbol{\Delta})$. In the phase plot, a - c: indicate the APFT, the peak of $A P$, and the peak of AHP, respectively; $\alpha \& \omega$ indicate maximal upstroke velocity, e.g. total inward currents and downstroke velocity, e.g. total outward currents, $(\boldsymbol{\Delta})$ indicates the location of the repolarization hump. Scale bar $=25 \mu \mathrm{m}$. 
Table 3: Microscopic, electrophysiological, and chemical criteria to differentiate subtypes of neurons isolated from nodose ganglia of neonatal and adult rats. Experiments were performed at room temperature $\left(\sim 22^{\circ} \mathrm{C}\right)$ and all data were average. Pooled data were presented as mean \pm I SD, $\mathrm{n}=$ number of tested neurons. $* P<0.05$ and $* * P<0.01$ vs A-types, $\dagger P<0.05$ and $\dagger \dagger P<0.0$ I vs $C$-types. $\# P<0.05$ and $\# P<0.0$ I by ANOVA among groups from either adult or neonate.

\begin{tabular}{|c|c|c|c|c|c|c|}
\hline \multirow[b]{2}{*}{ Type/n } & \multicolumn{3}{|c|}{ Adult ( 12 - 16 weeks) } & \multicolumn{3}{|c|}{ Neonate (5 - 11 days) } \\
\hline & A-type / 27 & Ah-type / 33 & C-type / 49 & A-type / 21 & Ah-type / 18 & C-type / 37 \\
\hline & \multicolumn{6}{|c|}{ Microscopic Criteria } \\
\hline Diameter $(\mu \mathrm{m})$ & $68.8 \pm 10.4$ & $59.4 \pm 8.4$ & $64.1 \pm 11.8$ & $47.6 \pm 5.67$ & $41.5 \pm 4.80$ & $43.9 \pm 8.6$ \\
\hline Longest Axis & $73.2 \pm 8.58$ & $62.4 \pm 6.44$ & $69.7 \pm 7.26$ & $52.1 \pm 9.33$ & $44.1 \pm 5.04$ & $49.9 \pm 11.2$ \\
\hline Shortest Axis & $52.3 \pm 6.84$ & $58.8 \pm 5.87$ & $59.5 \pm 5.91$ & $39.8 \pm 6.67$ & $41.6 \pm 3.25$ & $42.3 \pm 5.66$ \\
\hline Ratio \# & $0.71 \pm 0.29$ & $0.94 \pm 0.09^{* *} \dagger$ & $0.85 \pm 0.08$ & $0.76 \pm 0.22$ & $0.95 \pm 0.07^{*} \dagger$ & $0.83 \pm 0.11$ \\
\hline Membrane Roughness & NA & NA & NA & Entire surface & Central region & No \\
\hline Attached Fiber Diameter $(\mu \mathrm{m})$ & $6.12 \pm 1.34$ & $4.16 \pm 0.68^{*}$ & No & NA & NA & NA \\
\hline \multirow[t]{2}{*}{ Nucleus } & periphery & Unclear & $\sim$ Center & periphery & Unclear & $\sim$ Center \\
\hline & \multicolumn{6}{|c|}{ Electrophysiological Criteria } \\
\hline APFT (mV) \# & $-48.3 \pm 4.38$ & $-42.7 \pm 5.01 \dagger \dagger$ & $-31.3 \pm 3.92$ & $43.2 \pm 2.84$ & $-39.5 \pm 3.05 t \dagger$ & $-28.6 \pm 4.22$ \\
\hline UVMAX (V/s) \#\# & $389 \pm 34$ & $305 \pm 26^{* *}+\dagger$ & $164 \pm 27$ & $342 \pm 29$ & $297 \pm 23^{*}+\dagger$ & $152 \pm 19$ \\
\hline APD50 (ms) \#\# & $0.71 \pm 0.18$ & $1.87 \pm 0.35^{* *}+\dagger$ & $2.88 \pm 0.62$ & $0.92 \pm 0.24$ & $2.08 \pm 0.64^{*} \dagger$ & $3.22 \pm 0.78$ \\
\hline \multirow[t]{2}{*}{ Hump } & $(-)$ & $(+)$ & $(++)$ & $(-)$ & $(+)$ & $(++)$ \\
\hline & \multicolumn{6}{|c|}{ Chemosensitivity } \\
\hline$\alpha \beta$-m-ATP & $(+)$ & $(+)$ & $(-)$ & $(+)$ & $(+)$ & $(-)$ \\
\hline Capsaicin & $(-)$ & $(-)$ & $(+)$ & $(-)$ & $(-)$ & $(+)$ \\
\hline
\end{tabular}

Note: NA: Not applicable; $n$ : number of neurons; APFT: action potential firing threshold; UV MAx: maximal up-stroke velocity; APD $_{50}$ : action potential measured at $50 \%$ of height.

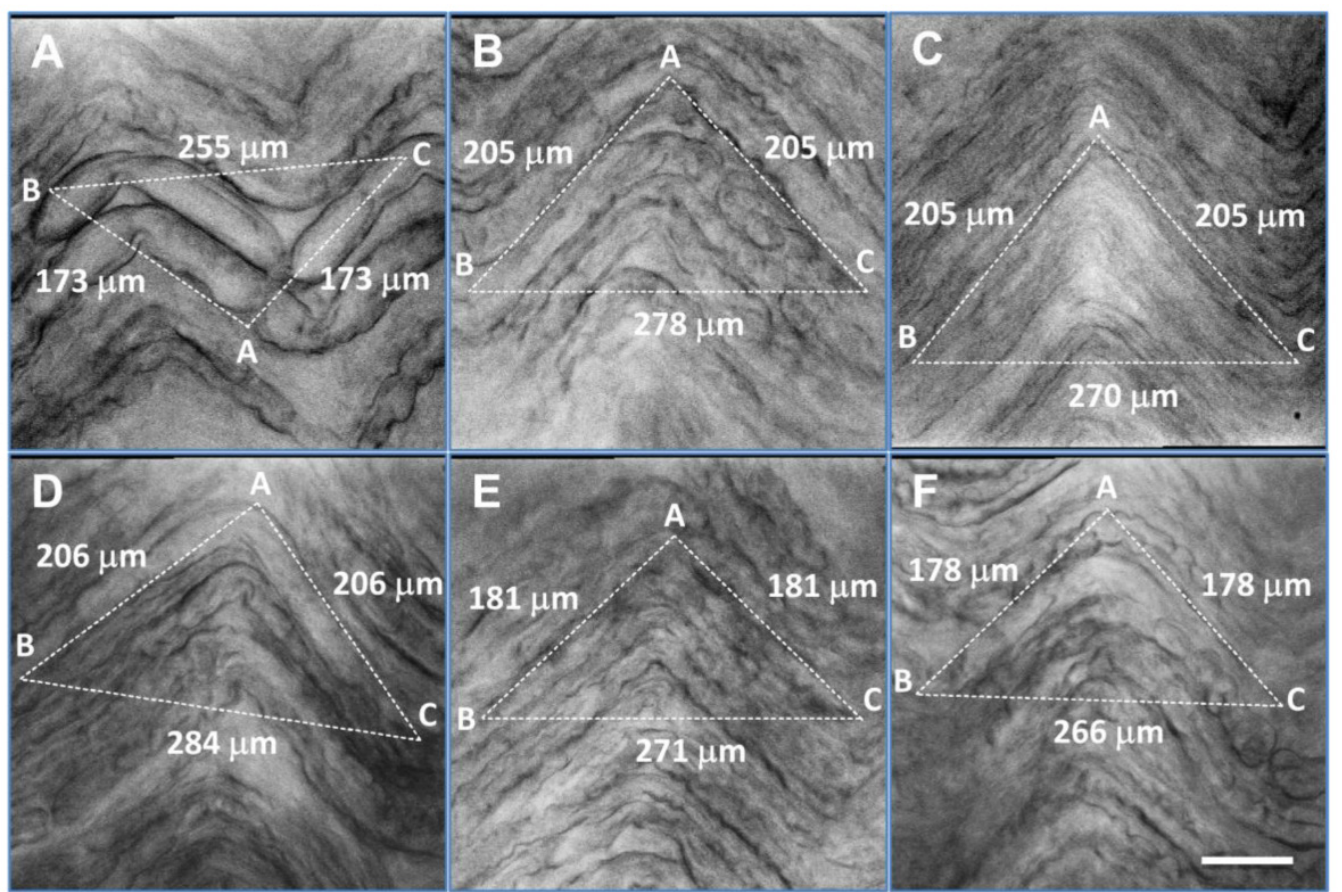

Figure 5: Distortion of afferent fibers after enzyme treatment and correction of calculated afferent conduction velocity. A - F: six representative examples of visceral afferent fibers from the vagus nerve after enzyme treatment. Large, myelinated fibers (A), regular-size myelinated fibers (B, D-F) and unmyelinated fibers $(C)$ exhibited marked shrinkage. Correction factors (ratio $=B C / A B+A C$ from dot line triangle, in which $A B$ and $A C$ were equal length and set to parallel with the fiber direction) were $0.73,0.68,0.66,0.69,0.75$, and 0.75 , respectively, for $(A)-(F)$. Scale bar $=35 \mu \mathrm{m}$. 
Table 4: Assessment of visceral afferent conduction velocity using a shrinkage correction factor. Bright field images of afferent fibers were taken and a triangle was superimposed on the nerve images such that two of its edges $(A B \& A C)$ were in paralleled with a fiber, while the third dot line $(B C)$ was set to close the triangle. $A B$ $+A C=$ estimated actual length of axon, and $B C=$ estimated axon length after enzyme treatment. The correction factor should be $B C$ divided by $(A B+A C)$. Average data are presented as mean \pm 1 $\mathrm{SD}, n=12$ images collected from separate slice preparations. $* * P<$ 0.001 vs before enzyme treatment.

\begin{tabular}{llll}
\hline Observation & $\begin{array}{l}\text { CV } \\
(\mathrm{m} / \mathrm{sec})\end{array}$ & $\begin{array}{l}\text { Calibration } \\
\text { Factor }\end{array}$ & $\begin{array}{l}\text { Correction } \\
(\mathrm{m} / \mathrm{sec})\end{array}$ \\
\hline Slice \#1 & 11.26, Figure 5D & 0.69 & 16.32 \\
Slice \#2 & 14.56, Figure 5E & 0.75 & 19.41 \\
Slice \#3 & 15.24, Figure 5A & 0.73 & 19.79 \\
Slice \#4 & 8.94 & 0.71 & 12.59 \\
Slice \#5 & 10.88 & 0.72 & 15.11 \\
Slice \#6 & 14.04 & 0.70 & 20.06 \\
Slice \#7 & 12.50, Figure 5C & 0.66 & 16.23 \\
Slice \#8 & 9.78 & 0.66 & 14.82 \\
Slice \#9 & 11.38, Figure 5B & 0.68 & 16.74 \\
Slice \#10 & 13.41 & 0.78 & 17.19 \\
Slice \#11 & 11.05 & 0.71 & 15.56 \\
Slice \#12 & 12.71, Figure 5F & 0.75 & 16.95 \\
Mean \pm SD & $12.15 \pm 1.85$ & $0.716 \pm 0.038$ & $16.94 \pm 2.18^{* *}$ \\
\hline
\end{tabular}

\section{Identification of neuron subtypes in nodose ganglia based on microscopic criteria}

Identification of myelinated Ah-type visceral afferent neurons as well as gender-specific Ah-type baroreceptor neurons in female rats is largely based on electrophysiological properties as determined previously in slice preparations (8). Ion channel and transporter expression profiles are not available for these neurons, nor are their responses to treatment with a variety of channel modulators. Accordingly, our foreseeable goal will be to gain insight into the molecular identities of ion transporting proteins and their functional modification by agonists and antagonists. Due to the experimental limitations of the slice preparation, isolated nodose neurons will be the preferred platform to accomplish these goals. Positive identification of Ah-type neurons in isolated cell preparations, however, will remain challenging. In our hands, direct comparisons of conduction velocity measurements in slice preparations as well as current responses to $\alpha \beta$-methyl-ATP or Cap application in isolated preparations with microscopic structural properties of nodose ganglia neurons enabled sensitive and specific identification of individual neurons under study. The criteria that were used to categorize neuron subtypes are listed in Table 3 . The positive predictive value of microscopic markers combined for identification of A-, C- and Ah-type neurons was
$90 \%, 98 \%$, and $\sim 76 \%$, respectively (Table 5). Only 7 out of 73 A-type neurons were incorrectly labeled as Ah-type neurons, and only 2 / 112 C-type neurons were misdiagnosed as Ah-types, whereas 20 / 85 Ah-type neurons were incorrectly classified as either A- or C-type neurons. These results support the notion that microscopic criteria can aid in identifying subtypes of neurons isolated from nodose ganglia. These observations also suggest that Ah-type neurons share the faster conduction and upstroke velocity with A-types, and also share the presence of a repolarization hump with C-type neurons. The repolarization hump reflects the activities of TTX-R Na+ currents (Supplementary Material: Figure S3B) $(1,11)$ and BK-type $\mathrm{Ca}^{2+}$-activated $\mathrm{K}^{+}$currents $(14,33)$ are typically absent in myelinated A-type neurons. Because of this specific feature of myelinated Ah-type visceral afferent neurons, extra attention has to be paid to distinguish it from either A- or C-types when $\mathrm{CV}$ measurements are not available for classification.

Table 5: Predictive value of microscopic structural properties for identification of neuronal subtypes in nodose ganglia from adult female rats. Observations are from 10 independent preparations. Electrophysiological measurements were compared with microscopic structural features.

\begin{tabular}{|c|c|c|c|c|c|}
\hline & \multicolumn{3}{|c|}{$\begin{array}{l}\text { Microscopic } \\
\text { identification }\end{array}$} & $\begin{array}{l}\text { Electrophysiological } \\
\text { identification }\end{array}$ & \multirow{2}{*}{$\begin{array}{l}\text { Positive } \\
\text { predictive } \\
\text { Value } \\
\%\end{array}$} \\
\hline & A-type & Ah-type & C-type & $n$ & \\
\hline A-type & 66 & 7 & 0 & 73 & $\begin{array}{l}90.04 \\
(66 / 73)\end{array}$ \\
\hline Ah-type & 8 & 65 & 12 & 85 & $\begin{array}{l}76.47 \\
(65 / 85)\end{array}$ \\
\hline C-type & 0 & 2 & 110 & 112 & $\begin{array}{l}98.21 \\
(110 / 112)\end{array}$ \\
\hline Overall & & & & 270 & $\begin{array}{l}89.26 \\
(214 / 270)\end{array}$ \\
\hline
\end{tabular}

$n=$ number of cells. Results were obtained from a total of 10 ganglia. Action potentials evoked by vagal nerve stimulation and direct current injection into the soma were analyzed. In some cases, the inward current responded to $\alpha \beta$-methyl-ATP $(100 \mathrm{nM})$ or capsaicin $(100 \mathrm{nM})$ was examined.

\section{Conclusion}

Here, we presented practical guidelines based on microscopic features to distinguish myelinated Ah-type neurons from myelinated A- and unmyelinated C-type nodose neurons for electrophysiological studies, and have reviewed advantages and disadvantages of isolated neuron and sliced ganglion preparations. Together, these observations should significantly facilitate future attempts to fully characterize the functional and molecular heterogeneity of nodose ganglia which play a key role in the regulation of cardiovascular, pulmonary, and gastrointestinal homeostasis. 


\section{Supplementary Material}

Fig.S1 - S3.

http://www.ijbs.com/v09p0716s1.pdf

\section{Acknowledgement}

Authors would like to thank Dr. Rubart-von der Lohe, Michael at Riley Heart Research Center, Wells Center for Pediatric Research, Indiana University, and School of Medicine for his valuable recommendation and prove readings.

This project was supported by research grants from Chinese Natural Science Foundation (№: 30973532; 81173051; 31171122) to G-F Q \& B-Y L, and partially supported by research grant from Education Department of China (№: 20112307110008) to B-Y L.

\section{Competing Interests}

The authors have declared that no competing interest exists.

\section{References}

1. Li BY, Schild JH. Electrophysiological and pharmacological validation of vagal afferent fiber type of neurons enzymatically isolated from rat nodose ganglia. J Neurosci Methods. 2007; 164: 75-85.

2. Li BY, Qiao GF, Feng B, Zhao RB, Lu YJ, Schild JH. Electrophysiological and neuroanatomical evidence of sexual dimorphism in aortic baroreceptor and vagal afferents in rat. Am J Physiol Regul Integr Comp Physiol. 2008; 295: R1301-10.

3. Kosta V, Guic MM, Aljinovic J, Caric A, Grkovic I. The influence of exercise on morphological and neurochemical properties of neurons in rat nodose ganglia. Neurosci Lett. 2011; 490: 36-40.

4. Kinch DC, Peters JH, Simasko SM. Comparative pharmacology of cholecystokinin induced activation of cultured vagal afferent neurons from rats and mice. PloS One. 2012; 7: e34755.

5. $\mathrm{Li}$ BY, Schild JH. Comparisons of somatic action potentials from dispersed and intact rat nodose ganglia using patch-clamp technique. Acta Pharmacol Sin. 2002; 23: 481-9.

6. Doan TN, Kunze DL. Contribution of the hyperpolarization-activated current to the resting membrane potential of rat nodose sensory neurons. J Physiol. 1999; 514: 125-38.

7. Qiao GF, Yang BF, Li WH, Li BY. Effects of artemisinin on action potentials from C-type nodose ganglion neurons. Acta Pharmacol Sin. 2003; 24: 937-42.

8. Li BY, Schild JH. Patch clamp electrophysiology in nodose ganglia of adult rat. J Neurosci Methods. 2002; 115: 157-67.

9. Schild JH, Clark JW, Hay M, Mendelowitz D, Andresen MC, Kunze DL. A- and C-type rat nodose sensory neurons: model interpretations of dynamic discharge characteristics. J Neurophysiol. 1994; 71: 2338-58.

10. Qiao GF, Li BY, Zhou YH, Lu YJ, Schild JH. Characterization of persistent TTX-R Na+ currents in physiological concentration of sodium in rat visceral afferents. Int J Biol Sci. 2009; 5: 293-7.

11. $\mathrm{Li} \mathrm{B}$, Schild JH. Persistent tetrodotoxin-resistant $\mathrm{Na}+$ currents are activated by prostaglandin E2 via cyclic AMP-dependent pathway in C-type nodose neurons of adult rats. Biochem Biophys Res Commun. 2007; 355: 1064-8

12. Kosta V, Guic MM, Aljinovic J, Sapunar D, Grkovic I. Immunohistochemical characteristics of neurons in nodose ganglia projecting to the different chambers of the rat heart. Auton Neurosci. 2010;155:33-8.

13. $\mathrm{Yu} \mathrm{S}$, Gao G, Peterson BZ, Ouyang A. TRPA1 in mast cell activation-induced long-lasting mechanical hypersensitivity of vagal afferent C-fibers in guinea pig esophagus. Am J Physiol Gastrointest Liver Physiol. 2009;297:G34-42.

14. Li BY, Glazebrook P, Kunze DL, Schild JH. KCa1.1 channel contributes to cell excitability in unmyelinated but not myelinated rat vagal afferents. Am J Physiol Cell Physiol. 2011;300:C1393-403.

15. Zhou YH, Sun LH, Liu ZH, Bu G, Pang XP, Sun SC, et al. Functional impact of the hyperpolarization-activated current on the excitability of myelinated A-type vagal afferent neurons in the rat. Clin Expe Pharmacol Physiol. 2010;37:852-61.

16. Qiao GF Qian Z, Sun HL, Xu WX, Yan ZY, Liu Y, Zhou JY, Zhang HC, Wang LJ, Pan XD, Fu YL. Remodeling of hyperpolarization-activated current, Ih, in Ah-type visceral ganglion neurons following ovariectomy in adult rats. PloS one. 2013; DOI:10.1371journal.pone.0071184.

17. Fukuda J. Nerve cells of adult and aged mice grown in a monolayer culture: age-associated changes in morphological and physiological properties of dorsal root ganglion cells in vitro. Dev Neurosci. 1985; 7: 374-94.

18. Qiao GF, Cheng ZF, Huo R, Sui XH, Lu YJ, Li BY. GM1 ganglioside contributes to retain the neuronal conduction and neuronal excitability in visceral and baroreceptor afferents. J Neurochem. 2008; 106: 1637-45.

19. Benn SC, Costigan M, Tate S, Fitzgerald M, Woolf CJ. Developmental expression of the TTX-resistant voltage-gated sodium channels Nav1.8 (SNS) and Nav1.9 (SNS2) in primary sensory neurons. J Neurosci. 2001; 21: $6077-85$.

20. Qian Z, Qiao G. F., Li B. Y. Ontogeny of neuronal excitability of vagal ganglion neurons and its ion channel mechanisms. CNS Neurosci Ther. 2013; In Revision.

21. McFarlane S, Cooper E. Postnatal development of voltage-gated K currents on rat sympathetic neurons. J Neurophysiol. 1992; 67: 1291-300.

22. Kasparov S, Paton JF. Changes in baroreceptor vagal reflex performance in the developing rat. Pflugers Arch. 1997; 434: 438-44.

23. Waki H, Yamasaki M, Katahira K, Katsuda S, Maeda M, Shimizu T. Developmental changes in functional characteristics of aortic baroreceptor afferents in rats. Exp Physiol. 2008; 93: 319-24.

24. Tu H, Liu J, Zhu Z, Zhang L, Pipinos II, Li YL. Mitochondria-derived superoxide and voltage-gated sodium channels in baroreceptor neurons from chronic heart-failure rats. J Neurophysiol. 2012; 107: 591-602.

25. Li YL. Elevated angiotensin II in rat nodose ganglia primes diabetes-blunted arterial baroreflex sensitivity: involvement of NADPH oxidase-derived superoxide. J Diabetes Metab. 2011; 2.

26. Vermehren-Schmaedick A, Jenkins VK, Hsieh HY, Brown AL, Page MP, Brooks VL, et al. Upregulation of brain-derived neurotrophic factor expression in nodose ganglia and the lower brainstem of hypertensive rats. J Neurosci Res. 2013; 91: 220-9.

27. Hanani M. Satellite glial cells in sensory ganglia: from form to function. Brain Res Rev. 2005; 48: 457-76.

28. Schild JH, Kunze DL. Differential distribution of voltage-gated channels in myelinated and unmyelinated baroreceptor afferents. Auton Neurosci. 2012; 172: 4-12.

29. Kollarik M, Dinh QT, Fischer A, Undem BJ. Capsaicin-sensitive and -insensitive vagal bronchopulmonary C-fibres in the mouse. J Physiol. 2003; 551: 869-79.

30. Yu S, Ru F, Ouyang A, Kollarik M. 5-Hydroxytryptamine selectively activates the vagal nodose C-fibre subtype in the guinea-pig oesophagus. Neurogastroenterol Motil. 2008; 20: 1042-50.

31. Zhang JM, Donnelly DF, LaMotte RH. Patch clamp recording from the intact dorsal root ganglion. J Neurosci Methods. 1998; 79: 97-103.

32. Fan W, Andresen MC. Differential frequency-dependent reflex integration of myelinated and nonmyelinated rat aortic baroreceptors. Am J Physiol. 1998; 275: H632-40.

33. Li BY, Feng B, Tsu HY, Schild JH. Unmyelinated visceral afferents exhibit frequency dependent action potential broadening while myelinated visceral afferents do not. Neurosci Lett. 2007; 421: 62-6. 